\title{
ISOLATION AND HIGH-PERFORMANCE THIN-LAYER CHROMATOGRAPHY ANALYSIS OF CONDITIONAL AMINO ACIDS FROM THE FRESH LEAVES OF ALTERNANTHERA SESSILIS
}

\author{
SWARNAKUMARI S*, MOHAN S, IDA CHRISTI VE, ABINAYA S, KALAIVANI S \\ Department of Pharmacognosy, Karpagam College of Pharmacy, Coimbatore, Tamil Nadu, India. Email: swarnapremanand@gmail.com
}

Received: 25 July 2018, Revised and Accepted: 03 October 2018

ABSTRACT

Objective: The objective of the study was to isolate the conditional amino acids present in the fresh leaves extracts of Alternanthera sessilis and compare the isolated amino acids with amino acid standards.

Method: The fresh plant material was used for extraction. For the extracted sample solution, paper chromatography and high-performance thin-layer chromatography (HPTLC) fingerprinting analysis were then carried out.

Results: Since A sessilis is rich in protein, various amino acids have been isolated in aggregates and paper chromatography has been performed. Amino acids were separated and identified using trial and error method. Cysteine, glycine, aspartic acid, and proline are the standard amino acids used for comparing with the isolated amino acids by HPTLC fingerprint analysis, which gives an idea for the authentication of the plant, its constituents and also provides a parameter for quality control.

Conclusion: The results scientifically validate the use of $A$. sessilis in the traditional medicine and also as a food.

Keywords: Fresh leaves extract, Alternanthera sessilis, Amino acids, High-performance thin-layer chromatography, Traditional medicine.

(C) 2019 The Authors. Published by Innovare Academic Sciences Pvt Ltd. This is an open access article under the CC BY license (http://creativecommons. org/licenses/by/4. 0/) DOI: http://dx.doi.org/10.22159/ajpcr.2019.v12i2.28728

\section{INTRODUCTION}

Metabolites are intermediate metabolic product and are of two types - primary and secondary. Primary metabolites usually perform intrinsic function and so present in all plants. Some common examples are carbohydrates, ethanol, lipids, nucleic acid, and proteins. Secondary metabolites usually not directly involved growth, development or reproduction but involved in defense function. Some common examples are alkaloids, terpenoids, glycosides, antibiotics, pigments, and plant growth regulators [1].

\section{Primary metabolites}

Primary metabolites are regarded as essential due to their function. They are components or products of fundamental metabolic pathways or cycles such as glycolysis, the Krebs cycle, and the Calvin cycle. Apart from its basic function, they act as a precursor for secondary metabolites and also an end product. Some antibiotics use primary metabolite as precursors, such as actinomycin created from primary metabolite, tryptophan [2].

\section{Amino acid}

Amino acids are primary metabolite, occurs in plant both in the free state and as the basic units of proteins and other metabolites. Some 20 different amino acids are isolated from proteins while other occurs in a free state, isolated from plants and microorganisms. Plant and bacteria can synthesize all 20 amino acids whereas mammals can able to synthesize only about half of them. Amino acid those synthesized in mammals are considered to be non-essential amino acid and not needed in the diet, whereas the remainder amino acids are considered an essential amino acid must be obtained from food [3]. As per the WHO guidelines, essential amino acid recommended for adult $(70 \mathrm{~kg}$ ) as follows, Tryptophan $-7 \mathrm{mg} / \mathrm{g}$, Threonine $-27 \mathrm{mg} / \mathrm{g}$, Isoleucine - $25 \mathrm{mg} / \mathrm{g}$, Leucine - $55 \mathrm{mg} / \mathrm{g}$, Lysine - $51 \mathrm{mg} / \mathrm{g}$, methionine and cysteine - $25 \mathrm{mg} / \mathrm{g}$, phenylalanine and tyrosine - $47 \mathrm{mg} / \mathrm{g}$, valine - $32 \mathrm{mg} / \mathrm{g}$, and histidine - $18 \mathrm{mg} / \mathrm{g}$ [4].
Six other amino acids are considered conditionally essential in the human diet, meaning their synthesis can be limited under special pathophysiological conditions, such as prematurity in the infant or individuals in severe catabolic distress. They are arginine, cysteine, gylcine, glutamine, proline, and tyrosine. The recommended daily intakes for children aged 3 years and older are 10-20\% higher than adult levels, and those for infants can be as much as $150 \%$ higher in the $1^{\text {st }}$ year of life. Cysteine (or sulfur-containing amino acids), tyrosine (or aromatic amino acids), and arginine are always required by infants and growing children [3].

Glycine is not essential to the human diet, as it is biosynthesized in the body from the amino acid serine, which is, in turn, derived from 3-phosphoglycerate, but the metabolic capacity for glycine biosynthesis does not satisfy the need for collagen synthesis. Pharmaceutical grade glycine is produced for some pharmaceutical applications, such as intravenous injections, where the customer's purity requirements often exceed the minimum required under the USP grade designation [3].

Aspartic acid deficiency leads to a decrease in cellular energy. Reduces stamina and chronic fatigue are the issues associated with this deficiency. It may also lead to depression. Amino acids help in the removal of excess ammonia from the body so aspartic acid deficiency may result in the elevated ammonia levels in the blood. This condition is responsible for the damage to liver, brain, and nervous system. D-aspartic acid is the supplement, responsible to increase the levels of testosterone in men. It helps the men to become muscular faster. However, the excess use of D-aspartic acid results in various side effects on health [4].

Many secondary metabolites are biosynthetically derived from an amino acid (primary metabolites). Some examples are proline, hydroxyl proline, ornithine, and arginine act as a precursor in the production of secondary metabolites like alkaloids. Amino acids have lots of applications in bodybuilding functions in plants, mammals and also precursors for secondary metabolites (biomolecules) [2]. 
Herbs are usually rich in amino acid, one such plant is A. sessilis, and its phytochemical analysis reveals the presence of amino acid [5] and also possesses wound healing activity [6]. A. sessilis commonly known as sessile Joyweed is a perennial herb, often found in and near ponds, canals, and reservoirs reported $5 \%$ of protein. In traditional medicine, the whole plant is used internally and externally and used to treat diarrhea, skin diseases, night blindness, indigestion, and fever. A. sessilis is being widely used for cooking by people of Tamil Nadu and other Southern states of India.

\section{METHODS}

\section{Collection of plant material}

A. sessilis leaves were collected in and around in Coimbatore District, Tamil Nadu, India, and authenticated by Dr. C. Murugan, Scientist "D" and Head of office, T.N.A.U, Coimbatore, Tamil Nadu, India. Authentication number BSI/SRC/5/23/2017/Tech-3527.

\section{Isolation of free amino acid}

Fresh leaves (150 mg) of A. sessilis were collected and kept in a frozen condition along with the liquid nitrogen to prevent degradation. Frozen leaves were grinded in a dry mortar along with liquid nitrogen and $600 \mu \mathrm{l}$ of a mixture of solutions containing water, chloroform, and methanol in a ratio $(3: 5: 12 \mathrm{v} / \mathrm{v})$. Addition of methanol makes the mixture homogenized. This homogenate is completely transferred to $2 \mathrm{ml}$ centrifuge tube and remaining in the mortar was also rinsed with mixture of solution and transferred to the centrifuge. Immediately the mixture was centrifuged at full speed for $2 \mathrm{~min}$ and supernatant solution was collected separately.

Re-extract the residue with another $600 \mu \mathrm{l}$ of mixture of solutions containing water, chloroform, and methanol for about 2 min and only the supernatant solution were transferred to fresh tube. Centrifugation continued till clear solution is obtained. Samples were stored at $-80^{\circ} \mathrm{C}$ and the extracted amino acids were quantified using ninhydrin assay. Usually, amino acid will be degraded within 1 week, so it is being quantified immediately [7].

This method is more reliable and less costly. Hence, free amino acid can be isolated from the fresh leaves of $A$. sessilis and can be used for further work.

\section{Paper chromatography}

Paper chromatography is an analytical method used to separate colored chemicals or substances. In paper chromatography, the sample mixture is applied to a piece of Whatman filter paper using the capillary tube and the edge of the paper is immersed in a solvent (mobile phase) and the solvent moves up the paper by capillary action. The filter paper is then dried and sprayed with ninhydrin reagent. Once again stationary phase dried in a hot air oven for $110^{\circ} \mathrm{C}$.

Amino acids isolated from fresh leaves are separated using chromatogram and compared with various standard amino acids using trial and error method [8].

Paper development: Ascending Techniques

Stationary phase: Whatman filter paper Grade 1

Mobile phase: n-Butanol:acetic acid:water (4:1:5)

Detecting agent: Ninhydrin reagent.

\section{High-performance thin-layer chromatography (HPTLC) of Amino acid}

HPTLC based methods are considered as a good alternative, as they are being explored as an important tool in routine drug analysis. Major advantage of HPTLC is its ability to analyze several samples simultaneously using a small quantity of mobile phase. This reduces time and cost of analysis. In addition, it minimizes exposure risks and significantly reduces disposal problems of toxic organic effluents, thereby reducing the possibilities of environment pollution. HPTLC also facilitates repeated detection of chromatogram with same or different parameters.
Cysteine, glycine, aspartic acid, and proline are four amino acids used as a standard, and the solution containing free amino acids isolated as per the method described above was used as a test solution for HPTLC analysis. n-butanol - $40 \%+$ glacial acetic acid - $10 \%+$ water - $50 \%$ was used as a solvent system to develop HPTLC fingerprint profile for amino acids containing the sample. $1 \mu \mathrm{l}$ of the standard solution and $2 \mu \mathrm{l}$ of test solution were loaded as $5 \mathrm{~mm}$ band length separately on precoated silica gel $60 \mathrm{~F} 254$ aluminum sheets $(3 \times 10 \mathrm{~cm})$ using a Hamilton syringe with the help of Linomat 5 applicator attached to a Camag HPTLC system, which was programmed through WIN CATS software. After the application of spots, the chromatogram was developed in twin trough glass chamber $(20 \times 10 \mathrm{~cm})$ pre-saturated with respective mobile phase. The air-dried plates were kept in a CAMAG Visualizer and images were captured at visible light, UV $366 \mathrm{~nm}$ and UV $254 \mathrm{~nm}$. The chromatograms were scanned by a CAMAG TLC Scanner after spraying with respective spray reagents and dried at $100^{\circ}$ in a hot air oven. The peak number with its height, area and $\mathrm{R} f$ values of fingerprint data were recorded by WIN CATS (1.3.4 version) software [9-14].

Stationary phase:

Plate size $(\mathrm{x} \times \mathrm{y}): 10.0 \times 10.0 \mathrm{~cm}$

Material: HPTLC plate silica gel 60 F 254

Manufacturer : E. MERCK KGaA

Sample application- CAMAG Linomat 5:

Instrument: CAMAG Linomat 5 "Linomat5_170147"

Linomat 5 Application Parameters

Spray gas : Inert gas

Sample solvent type: Methanol

Dosage speed : $150 \mathrm{nl} / \mathrm{s}$

Predosage volume: $0.2 \mathrm{ul}$

Sequence

Syringe size : 100 microliter

Number of tracks: 5

Application position $Y: 8.0 \mathrm{~mm}$

Band length : $8 \mathrm{~mm}$

Development-glass tank:

Chamber type : Twin Trough Chamber $10 \times 10 \mathrm{~cm}$

Executed by : AAS

Pre Conditioning

Mobile phase : n-Butanol 40\%: Glacial acetic acid 10\%: Water 50\%

Solvent front position: $70.0 \mathrm{~mm}$

Volume : $10.0 \mathrm{ml}$

Drying device : oven

Temperature: $60^{\circ} \mathrm{C}$

Time : 5 min

Detection

Instrument : CAMAG TLC Scanner

\section{RESULTS AND DISCUSSION}

Isolation of free amino acid

From fresh leaves juice of $A$. sessilis, as per the procedure discussed earlier various free amino acids are isolated in the form of aggregates. Hence, to separate the amino acids aggregates and also to assume the amino acid, paper chromatography method is applied.

\section{Paper chromatography}

Since paper chromatography is one of the reliable methods for determination of amino acid, isolated amino acids are identified using trial and error method. n-Butanol:glacial acetic acid:water in a ratio 4:5:1 is used as a mobile phase since polar side chain amino acids (aspartic acid, cysteine, lysine, etc.) and shorter non-polar amino acids (proline, alanine, and glycine) migrates faster in this solvent. The sample is separately developed and viewed at $362 \mathrm{~nm}$ reported in Fig. 1 and also viewed after spraying detecting agent (ninhydrin reagent) is reported in Fig. 2.

Then using trial and error method, the sample containing amino acid is being compared with various standard amino acids and four amino acids have been assumed based on the coinciding $\mathrm{R}_{\mathrm{f}}$ values. 


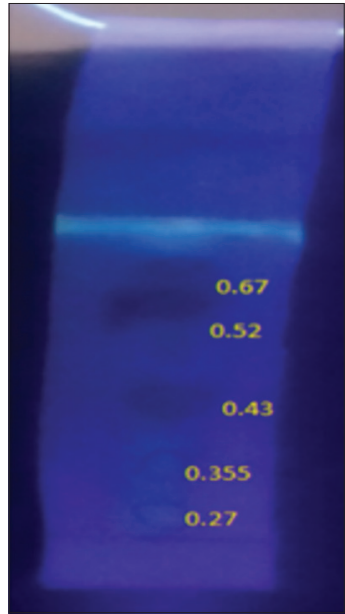

Fig. 1: Paper chromatography of isolated amino acid detected at UV 362nm

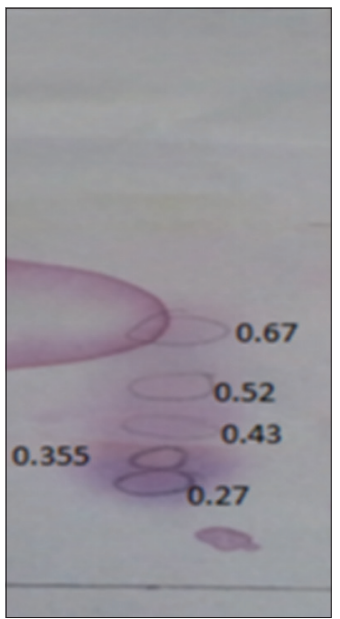

Fig. 2: Paper chromatography of isolated amino acid detected using ninhydrin

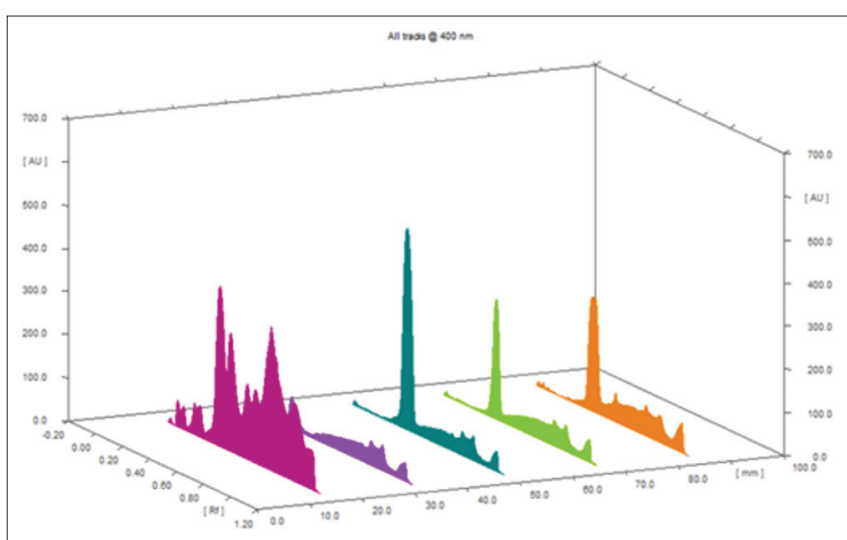

Fig. 3: 3- Dimensional view of chromatogram of sample with four standard amino acids

The amino acids assumed by paper chromatography are cysteine, glycine, aspartic acid, and proline.

\section{HPTLC analysis of amino acid}

Seven different types of amino acids were observed with peak value from the isolated sample of $A$. sessilis. The suitable solvent system selected for evaluation was n-Butanol:glacial acetic acid:water (4:1:5).

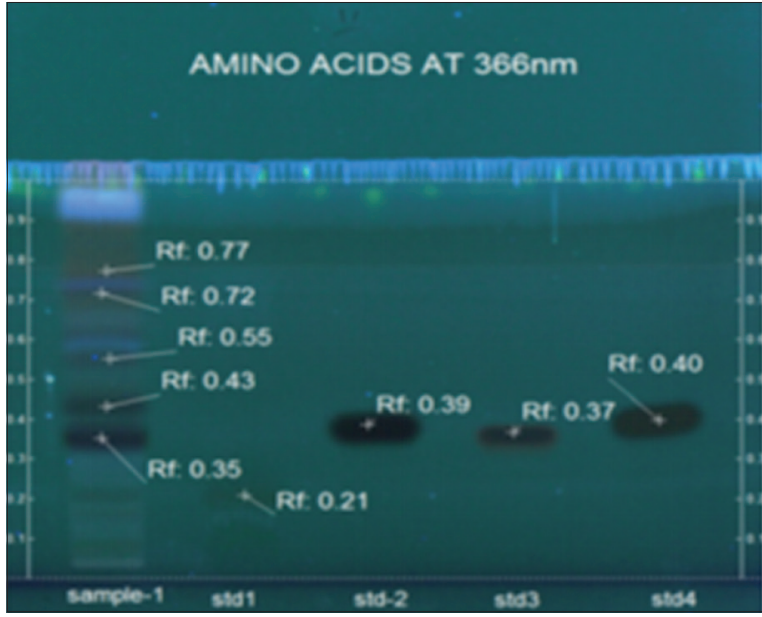

Fig. 4: High-performance thin-layer chromatography chromatogram of the amino acid at $366 \mathrm{~nm}$

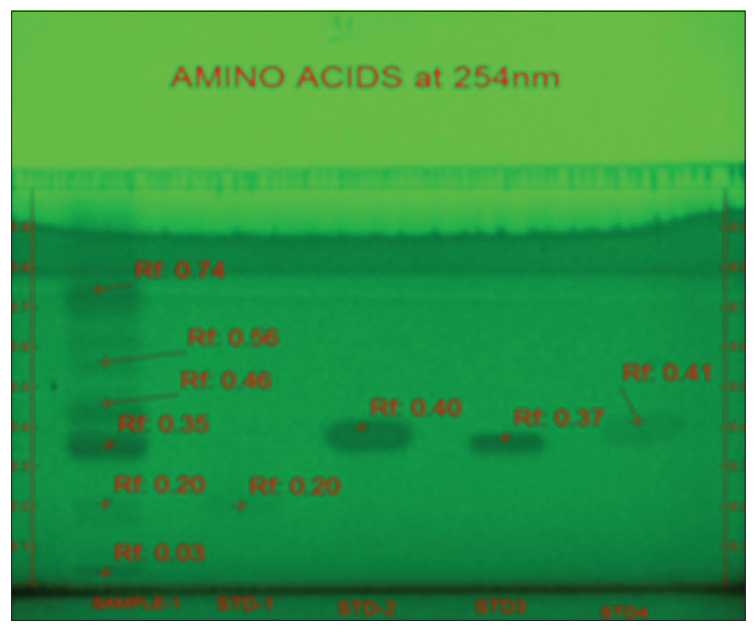

Fig. 5: High-performance thin-layer chromatography chromatogram of the amino acid at $254 \mathrm{~nm}$

The chromatogram of the sample was developed along with the four standard amino acids. HPTLC fingerprint of a plant species provides basic information regarding isolation, purification, and characterization. This present study provides sufficient information about amino acids present in fresh leaves of $A$. sessilis and also identification, standardization and quality control of the medicinal plant. HPTLC chromatogram of the sample was reported in Table 1. indicates the presence of seven compounds.

3- D dimension of sample with standard amino acid represented in Fig. 3. The results from HPTLC scanned at $366 \mathrm{~nm}$ and reported in Fig. 4, for the amino acid isolated from fresh leaves of $A$. sessilis, reveals the presence of 5 amino acids with the $R_{\mathrm{f}}$ values: $0.35,0.43,0.55,0.72$, and 0.77 . The $R_{\mathrm{f}}$ values for standard amino acids cysteine, glycine, aspartic acid, and proline are 0.21 , $0.39,0.37$, and 0.40 , respectively. Comparing with the four standard amino acids, the $R_{f}$ value of standard (I) 0.37 coincide with $R_{f}$ value of sample 0.35 and the $\mathrm{R}_{\mathrm{f}}$ value of standard (II) 0.40 coincide with $\mathrm{R}_{\mathrm{f}}$ value of sample 0.43 .

The results from HPTLC scanned at $254 \mathrm{~nm}$ and reported in Fig. 5, for an isolated sample containing amino acid reveals the presence of 6 amino acids with the $\mathrm{R}_{\mathrm{f}}$ values: $0.03,0.20,0.35,0.46,0.56$, and 0.74 . The $\mathrm{R}_{\mathrm{f}}$ values for standard amino acids cysteine, glycine, aspartic acid, and proline are $0.20,0.40,0.37$, and 0.41 , respectively. When comparing with the four standard amino acids, the $\mathrm{R}_{\mathrm{f}}$ value of standard (I) 0.20 coincide with $R_{f}$ value of sample 0.20 , the $R_{f}$ value of standard (II) 0.40 coincide with $R_{f}$ value of sample 0.35 , and the $R_{f}$ value of standard (III) 0.41 coincide with $\mathrm{R}_{\mathrm{f}}$ value of sample 0.46 . 


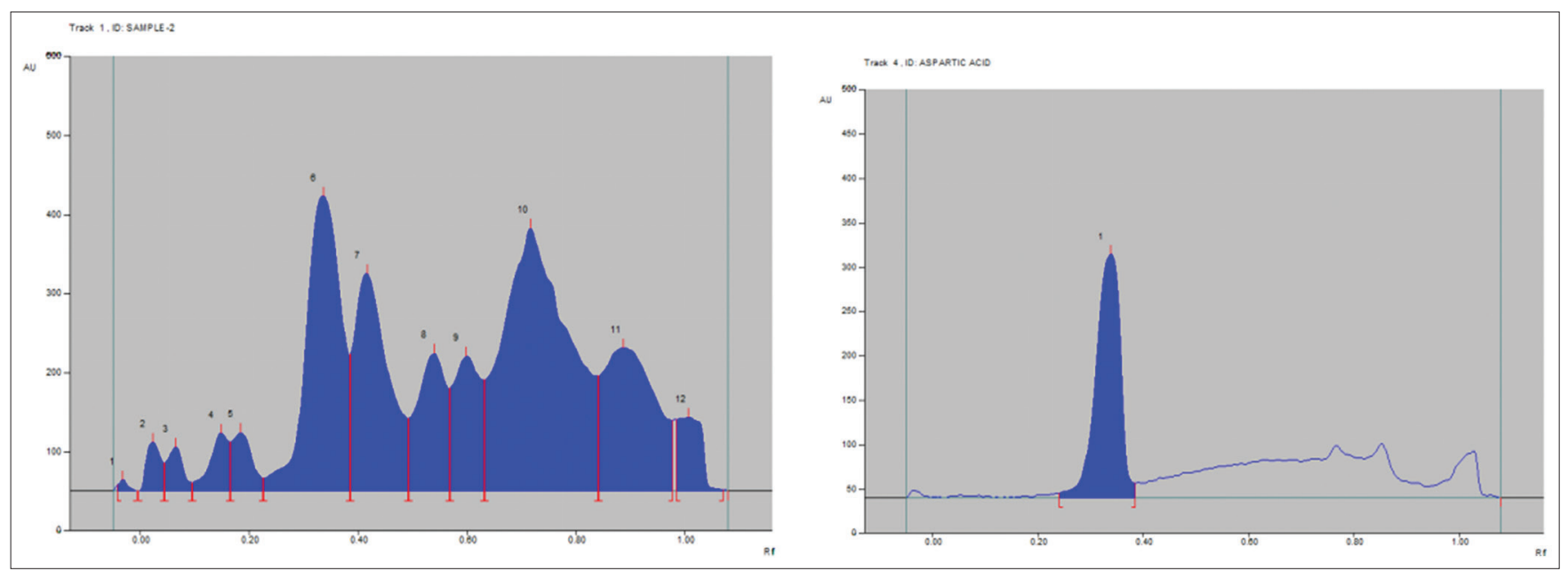

Fig. 6: Comparison of high-performance thin-layer chromatography fingerprint sample with standard amino acid (aspartic acid)

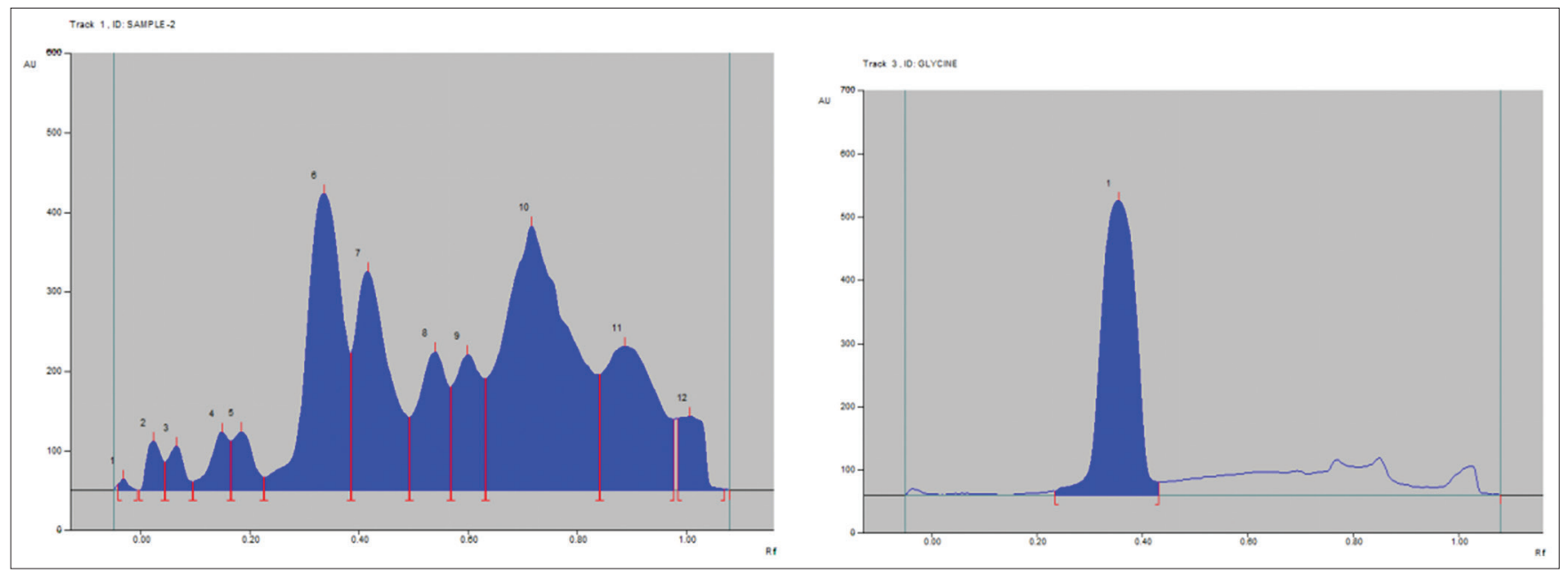

Fig. 7: Comparison of high-performance thin-layer chromatography fingerprint sample with standard amino acid (glycine)

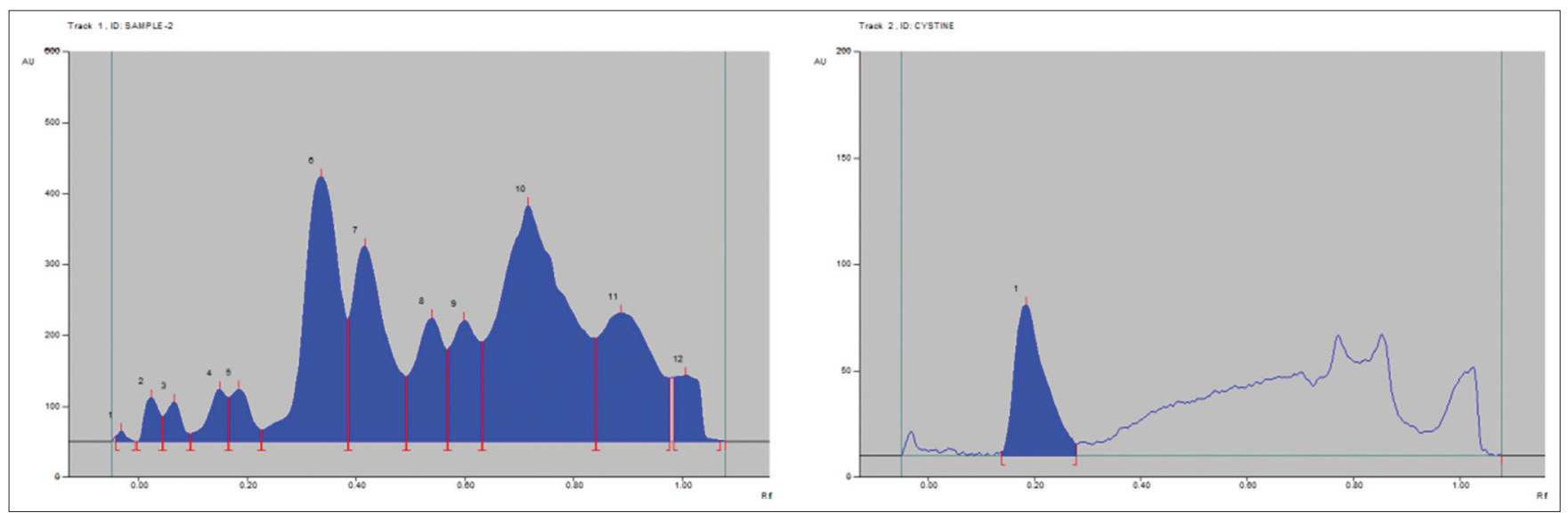

Fig. 8: Comparison of high-performance thin-layer chromatography fingerprint sample with standard amino acid (cysteine)

HPTLC chromatogram was developed; ninhydrin reagent was sprayed and observed. HPTLC fingerprint sample has been compared with standard amino acids - aspartic acid, glycine, cysteine, and proline and reported in Figs. 6-9.

Standard I - Cysteine

Standard II - Glycine

Standard III - Aspartic acid

Standard IV - Proline.

\section{DISCUSSION}

Mixture of amino acids has been successfully isolated from the fresh leaves of $A$. sessilis and assumed using paper chromatogram (trial and error method). Based on the paper chromatogram, standard amino acids are decided. From HPTLC, we can conclude the presence of amino acids such as cysteine, aspartic acid, and proline. Hence, plant or plant extract can be given in deficiency of these conditional amino 


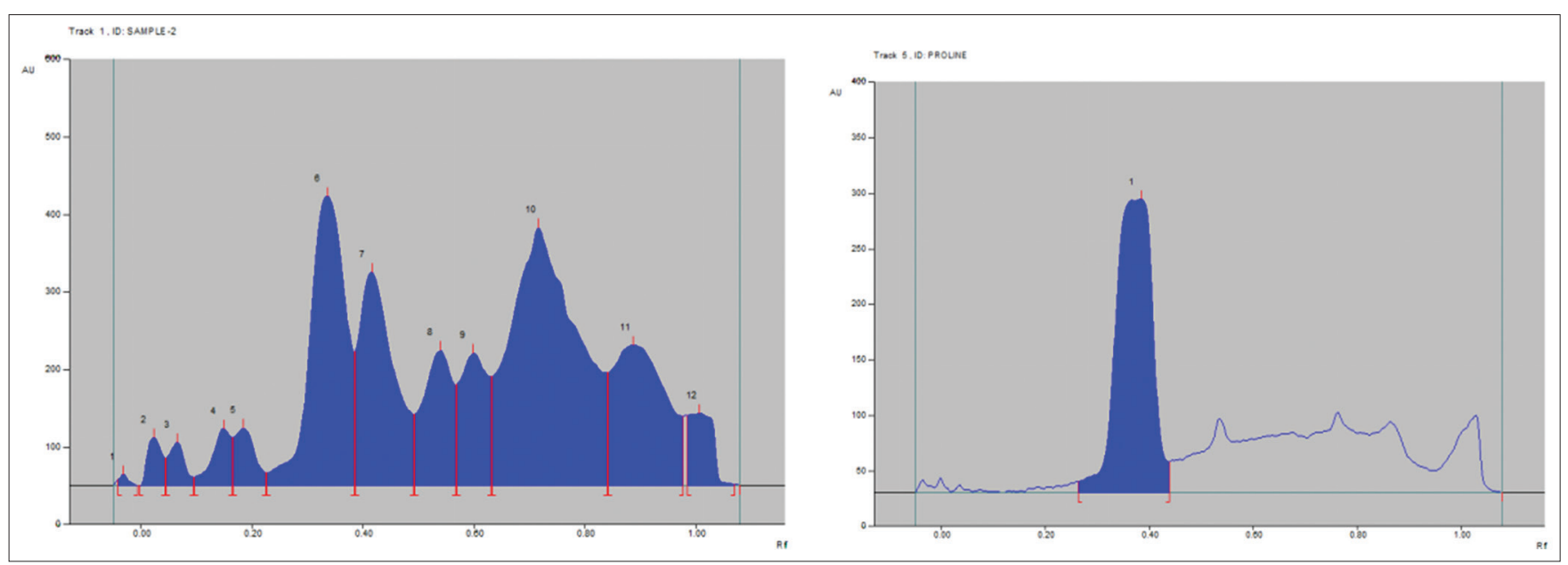

Fig. 9: Comparison of high-performance thin-layer chromatography fingerprint of sample with standard amino acid (proline)

Table 1: HPTLC profile for sample

\begin{tabular}{lll}
\hline S.No & R $_{\mathrm{f}}$ value & Peak area \\
\hline 1 & 0.34 & 17063.0 \\
2 & 0.42 & 12353.7 \\
3 & 0.54 & 6528.4 \\
4 & 0.60 & 5943.4 \\
5 & 0.72 & 28880.4 \\
6 & 0.89 & 12464.1 \\
7 & 1.01 & 2984.6 \\
\hline HPTLC: High-performance thin-layer chromatography
\end{tabular}

acids. The result obtained by qualitative evaluation of HPTLC will be helpful in the further quantitative estimation of each and every amino acid and also for standardization of herbal formulations containing this plant.

\section{CONCLUSION}

It can be concluded that based on the results obtained from the HPTLC fingerprint analysis will be helpful in identification and standardization of $A$. sessilis and can be utilized as a reference for the identification and quality control of the drug. The present study can be considered as reference and can be used for validation of the plant usage.

\section{ACKNOWLEDGMENT}

The authors thank the management of the college for encouraging and providing the research facilities. The authors also thank Dr. C. Murugan for identification of the plant and Dr. M. Sasikala for her enormous support during the completion of work.

\section{AUTHORS' CONTRIBUTION}

All authors have contributed equally to carry out the work.

\section{CONFLICTS OF INTEREST}

The authors declared that they have no conflicts of interest.

\section{REFERENCES}

1. Hussain MS, Fareed S, Ansari S, Rahman MA, Ahmad IZ, Saeed M, et al. Current approaches toward production of secondary plant metabolites. J Pharm Bioallied Sci 2012;4:10-20.

2. Irchhaiya R, Kumar A, Yadav A, Gupta N, Kumar S, Gupta N, et al. Metabolites in plants and its classification. World J Pharm Pharm Sci 2015;4:287-305.

3. Wang W, Wu Z, Dai Z, Yang Y, Wang J, Wu G, et al. Glycine metabolism in animals and humans: Implications for nutrition and health. Amino Acids 2013;45:463-77.

4. Azevedo RA, Lancien M, Lea PJ. The aspartic acid metabolic pathway, an exciting and essential pathway in plants. Amino Acids 2006;30:143-62.

5. Kumari EV, Krishnan V. Qualitative and quantitative estimation of phytochemicals of Alternanthera sessilis(L) R. Br. Ex. Dc and Alternanthera philoxeoides (Mart). Griseb. Int J Bot Res 2016;6:29-34.

6. Arun M, Satish S, Anima P. Herbal boon for wounds. Int J Pharm Pharm Sci 2013;5:1-12.

7. Carpena-Ruiz R, Sopena A, Ramon AM. Extraction of free amino acids from tomato leaves. Plant Soil 1989;119:251-4.

8. Iffat AM, Ahmed HM. Some aspects of solute concentration and zone area of the spot for detection and quantification of amino acids by paper chromatography. J Chem Soc Pak 1993;15:172-8.

9. Karthika K, Paulsamy S. TLC and HPTLC fingerprints of various secondary metabolites in the stem of the traditional medicinal climber, Solenaam plexicaulis. Indian J Pharm Sci 2015;77:111-6.

10. Nile SH, Park SW. HPTLC analysis, antioxidant and antigout activity of Indian plants. Iran J Pharm Res 2014;13:531-9.

11. Seasotiya L, Siwach P, Malik A, Bal S. Phytochemical evaluation and HPTLC fingerprint profile of Cassia fistula. Int J Adv Pharm Biol Chem 2014;3:604-11.

12. Shoaib A, Siddiqui HH, Badruddeen B, Rizvi A, Dixit RA. Physicochemical, phytochemical and high-performance thin layer chromatography analysis of the root barks of Onosma echioides. Asian J Pharm Clin Res 2017;10:196-9.

13. Gurupriya S, Cathrine L, Pratheema L. HPTLC method for the determination of lupeol from Andrographis echioides leaves. Int $\mathrm{J}$ Pharm Pharm Sci 2018;10:102-7.

14. Mritunjay K, Mondal DB, Ananya D. Quantification of catechin and lycophene in Calendula officinalis extract using HPTLC method. Asian J Pharm Clin Res 2011;4 Supp 2:128-9. 